\title{
Atenção farmacêutica a idosos portadores de doenças crônicas no âmbito da atenção primária à saúde
}

\author{
Pharmaceutical care for elderly with chronic diseases \\ at the primary health care
}

Recebido em: 05/10/2017

Aceito em: 10/04/2018

Edimar FERREIRA JÚNIOR; Almária Mariz BATISTA Departamento de Farmácia, Centro de Ciências da Saúde, Universidade Federal do Rio Grande do Norte. Av. General Gustavo Cordeiro de Farias, s/n, CEP 59010-180. Natal, RN, Brasil.E-mail: almariamariz@yahoo.com.br

\section{ABSTRACT}

Pharmaceutical Care is a practice developed in the context of Pharmaceutical Assistance, comprising attitudes, ethical values, behaviors, skills, commitments, and co-responsibilities in disease prevention, health promotion and recovery, in an integrated way to the health workers team. It focuses on the drug user, health education, pharmaceutical orientation, activities systematic recording, results in measurement and evaluation, in the search for a satisfactory response to the pharmacological therapy. The present study aimed to review the relevant literature on Pharmaceutical Care in the context of elderly with chronic diseases attended by primary health care. A bibliographic review was carried out, using the databases NCBI (via Pubmed), Web of Science, Scopus, and LILACS. Without the pharmacist performance, the medication and care related to its use are disorderly and disqualified at the SUS. There were significant problems at all points involving pharmacotherapy, such as adherence, polypharmacotherapy, self-medication, drug problems and drug interactions. Pharmaceutical Care in the context of elderly health problems is essential for the strengthening of adherence to treatment, expenses reduction, medicines rational use promotion, drug problems reduction, disease information.

Keywords: pharmaceutical care; primary health care; elderly; chronic disease

\section{RESUMO}

Atenção Farmacêutica constitui a prática farmacêutica desenvolvida no contexto da Assistência Farmacêutica, compreendendo atitudes, valores éticos, comportamentos, habilidades, compromissos e co-responsabilidades na prevenção de doenças, promoção e recuperação da saúde, de forma integrada à equipe de saúde. Tem foco no usuário do medicamento, na educação em saúde, na orientação farmacêutica, no registro sistemático de atividades, mensuração e avaliação de resultados, na busca da resposta satisfatória à terapêutica farmacológica. O presente estudo teve como objetivo revisar a literatura pertinente à Atenção Farmacêutica no contexto de idosos portadores de doenças crônicas atendidos pela atenção primária à saúde. Trata-se de revisão bibliográfica realizada nas bases de dados NCBI (via Pubmed), Web of Science, Scopus e LILACS. Sem a atuação do farmacêutico, o medicamento e o cuidado relacionado ao seu uso apresentam-se desordenados e desqualificados no âmbito do SUS. Há problemas significativos em todos os pontos envolvendo a farmacoterapia, como adesão, polifarmacoterapia, automedicação, problemas relacionados a medicamentos e interações medicamentosas. A Atenção Farmacêutica no contexto de problemas de saúde em idosos é essencial para o fortalecimento da adesão ao tratamento, redução dos gastos, promoção do uso racional dos medicamentos, redução dos problemas relacionados aos medicamentos, informações sobre a doença e seus agravos.

Palavras-chave: atenção farmacêutica; atenção primária à saúde; idoso; doença crônica 


\section{INTRODUÇÃO}

O Sistema Único de Saúde (SUS) tem mais de duas décadas de existência, sendo, atualmente, um dos maiores sistemas públicos de saúde do mundo e o único a garantir assistência integral e gratuita a toda população (1). A população brasileira com mais de 60 anos é de cerca de 21 milhões de pessoas, sendo aproximadamente 12 milhões do sexo feminino, com estimativa do dobro desta população idosa em 2030 (2).

O envelhecimento é um processo natural que traz alterações biopsicossociológicas únicas. As mudanças fisiológicas verificadas nos idosos refletem não só este processo, mas também os efeitos dos anos na exposição a agentes ambientais, como luz do sol, bebidas alcoólicas e fumo de cigarros, além de processos de doença, tais como diabetes mellitus, dislipidemias, hipertensão, hipotireoidismo e artrite (3).

Nesse contexto, a Atenção Primária à saúde caracteriza-se como conjunto de ações, nos âmbitos individual e coletivo, que abrange promoção, proteção e manutenção da saúde com objetivo de atenção integral que impacte na situação de saúde, autonomia das pessoas e determinantes/condicionantes de saúde das coletividades. Desenvolve-se via práticas de cuidado e gestão, democráticas e participativas, sob forma de trabalho em equipe, dirigidas a populações de territórios definidos, pelas quais assume responsabilidade sanitária, considerando a dinamicidade do território em que vivem (4).

A Assistência Farmacêutica, por sua vez, constitui conjunto de ações voltadas a promoção, proteção e recuperação da saúde, tanto individual como coletiva, tendo o medicamento como insumo essencial $\mathrm{e}$ visando seu acesso e uso racional. Envolve pesquisa, desenvolvimento e produção de medicamentos e insumos, bem como sua seleção, programação, aquisição, distribuição, dispensação, garantia da qualidade de produtos e serviços, acompanhamento e avaliação de sua utilização (5).

Isto posto, a Atenção Farmacêutica constitui prática farmacêutica desenvolvida no contexto da Assistência Farmacêutica, compreendendo atitudes, valores éticos, comportamentos, habilidades, compromissos e corresponsabilidades na prevenção de doenças, promoção e recuperação da saúde, de forma integrada à equipe de saúde. Interação direta entre farmacêutico e usuário, visando farmacoterapia racional e resultados definidos e mensuráveis, voltados a melhoria da qualidade de vida, devendo também envolver concepções de seus sujeitos, respeitadas suas especificidades bio-psico-sociais, sob a óptica da integralidade das ações de saúde (5).

Diante deste contexto, o presente estudo teve como objetivo revisar a literatura pertinente a Atenção Farmacêutica no contexto de idosos portadores de doenças crônicas atendidos em nível de Atenção Primária à saúde.

\section{MÉTODO}

Trata-se de revisão bibliográfica, cuja estratégia de busca foi a utilização dos descritores em ciências da saúde Atenção Primária à Saúde, Atenção Farmacêutica, Idoso e Doença Crônica, os quais foram submetidos às bases de dados National Center for Biotechnology Information (NCBI) (via Pubmed), Web of Science, Scopus e Literatura Latino-Americana e do Caribe em Ciências da Saúde (LILACS).

Após leitura flutuante de títulos e resumos, foram excluídos artigos repetidos e que não representavam a temática da atenção farmacêutica no âmbito da atenção primária à saúde.

Os critérios de inclusão estabelecidos foram artigos brasileiros publicados de 2000 a 2016, conforme Quadro 1 . 
Quadro 1: Artigos selecionados conforme a estratégia de busca adotada, a saber, publicações brasileiras sobre o tema, no período 2000-2016

\begin{tabular}{|c|c|}
\hline Artigo & Título \\
\hline$A$ & $\begin{array}{l}\text { Bortolon PC, Karnikowski MG0, Assis M. Automedicação versus indicação farmacêutica: o profissional de far- } \\
\text { mácia na atenção primária à saúde do idoso. Revista APS. } 2007 ; 10(2): 200-209 \text {. }\end{array}$ \\
\hline B & $\begin{array}{l}\text { Fernandes WS, Cembranelli JC. Automedicação e o uso irracional de medicamentos: o papel do profissional } \\
\text { farmacêutico no combate a essas práticas. Rev. Univap. 2015;21(37):05-12. D0I: 10.18066/revistaunivap. } \\
\text { v21i37.265. }\end{array}$ \\
\hline C & $\begin{array}{l}\text { Sá M, Barros JAC, Sá MPB. 0. Automedicação em idosos na cidade de Salgueiro-PE. Rev. bras. epidemiol. } \\
\text { 2007;10(1):75-85. DOI: 10.1590/S1415-790X2007000100009. }\end{array}$ \\
\hline$D$ & $\begin{array}{l}\text { Santos TRA, Lima DM, Nakatani AYK, Pereira LV, Leal GS, Amaral RG. Consumo de medicamentos por idosos. } \\
\text { Rev. Saúde Pública. 2013; 47(1):94-103. DOI: 10.1590/S0034-89102013000100013. }\end{array}$ \\
\hline$E$ & $\begin{array}{l}\text { Lima-Costa MF, Firmo J0A, Uchoa EA. A estrutura da autoavaliação da saúde entre idosos: projeto Bambuí. } \\
\text { Rev Saúde Pública. 2004;38(6):827-34. DOI: 10.1590/S0034-89102004000600011. }\end{array}$ \\
\hline$F$ & $\begin{array}{l}\text { Silveira EA, Dalastra L, Pagotto V. Polifarmácia, doenças crônicas e marcadores nutricionais em idosos. Rev. } \\
\text { Bras. Epidemiol. 2014;17(4):818-829. DOI: 10.1590/1809-4503201400040002. }\end{array}$ \\
\hline$G$ & $\begin{array}{l}\text { Alano GM, Corrêa TS, Galato D. Indicadores do serviço de atenção farmacêutica (SAF) da Universidade do Sul } \\
\text { de Santa Catarina. Ciência \& Saúde coletiva. 2012;17(3):757-764. DOI: 10.1590/S1413-81232012000300023. }\end{array}$ \\
\hline $\mathrm{H}$ & $\begin{array}{l}\text { Provin MP, Campos AP, Neilson SE0, Amaral RG. Atenção farmacêutica em Goiânia: inserção do farmacêutico } \\
\text { na estratégia saúde da família. Saúde Soc. 2010;19(3):717-723. DOI: 10.1590/S0104-12902010000300022. }\end{array}$ \\
\hline I & $\begin{array}{l}\text { Foppa AA, Bevilacqua G, Pinto HL, Blatt CR. Atenção farmacêutica no contexto da estratégia de saúde da } \\
\text { família. Rev. Bras. Cien. Farm. 2008;44(4): 728-737. DOI: 10.1590/S1516-93322008000400020. }\end{array}$ \\
\hline $\mathrm{J}$ & $\begin{array}{l}\text { Amaral DMD, Perassolo MS. Possíveis interações medicamentosas entre os anti-hipertensivos e antidiabé- } \\
\text { ticos em participantes do Grupo HIPERDIA de Perobé, RS (Uma análise teórica). Rev. Farm. Básica e Apl. } \\
\text { 2013;33(1):99-105. }\end{array}$ \\
\hline K & $\begin{array}{l}\text { Correr CJ, Pontarolo R, Ferreira LC, Baptistão SAM. Risco de problemas relacionados com medicamentos em } \\
\text { pacientes de uma instituição geriátrica. Rev. Bras. Ciênc. Farm. 2007;43(1):56-62. DOI: 10.1590/S1516- } \\
93322007000100007 \text {. }\end{array}$ \\
\hline $\mathrm{L}$ & $\begin{array}{l}\text { Obreli-Neto PR, Prado MF, Vieira JC, Pelloso SM, Marcon SS, Cuman RKN. Fatores interferentes na taxa de ade- } \\
\text { são à farmacoterapia em idosos atendidos na rede pública de saúde do Município de Salto Grande-SP, Brasil. } \\
\text { Rev. Ciênc. Farm. Básica e Apl. 2010;31(3):229-233. }\end{array}$ \\
\hline$M$ & $\begin{array}{l}\text { Sardinha AHL, Silva CG, Sena LB, Mesquita LLS, Rodrigues JB, Silva KNR. Adesão dos idosos com doenças } \\
\text { crônicas ao tratamento medicamentoso. Rev. Pesq. Saúde. 2015;16(3):154-158. }\end{array}$ \\
\hline $\mathrm{N}$ & $\begin{array}{l}\text { Landim MP, Oliveira CJ, Abreu RNDC, Moreira TMM, Vasconcelos SMM. Adesão ao tratamento farmacológico } \\
\text { anti-hipertensivo por pacientes de unidade da estratégia saúde da família. Rev. APS. 2011;14(2):132-138. }\end{array}$ \\
\hline
\end{tabular}

\section{RESULTADOS E DISCUSSÃO}

Perfil Demográfico e Socioeconômico. Na Tabela 1 pode ser observada a predominância do gênero feminino (69\%), a maior tendência ao autocuidado em relação aos homens, além do espírito de matriarca e cuidadora do lar e família. Ainda, mostra o acompanhamento de cuidador, podendo ser familiar ou não, notando-se que a maioria dos idosos mora acompanhado (72\%). Entretanto, isto não significa que a farmacoterapia será efetiva e segura para o idoso,

Quanto à renda mensal, 50\% recebiam 1 salário mínimo, sendo aposentados e trabalhadores de baixa renda. Tal fato pode interferir na farmacoterapia, devido à dificuldade de aquisição dos medicamentos, considerando o limitado acesso ao medicamento no serviço de saúde público, ou não adequado para suprir a população. 
Tabela 1. Classificação socio-econômica e demográfica de idosos, de acordo com a literatura consultada (20002016)

\begin{tabular}{|c|c|}
\hline \multicolumn{2}{|l|}{ Gênero } \\
\hline Feminino & $69 \%$ \\
\hline Masculino & $31 \%$ \\
\hline \multicolumn{2}{|l|}{ Renda mensal } \\
\hline 1 salário mínimo & $50 \%$ \\
\hline 2 salários mínimos & $30 \%$ \\
\hline 3 salários mínimos & $15 \%$ \\
\hline > 4 salários mínimos & $5 \%$ \\
\hline \multicolumn{2}{|l|}{ Escolaridade } \\
\hline Analfabetos & $27 \%$ \\
\hline Ensino fundamental iniciado/completo & $46 \%$ \\
\hline Ensino médio iniciado/completo & $21 \%$ \\
\hline Ensino superior & $6 \%$ \\
\hline \multicolumn{2}{|c|}{ Presença de acompanhante em casa } \\
\hline Sim & $72 \%$ \\
\hline Não & $28 \%$ \\
\hline
\end{tabular}

Outro ponto que pode interferir na farmacoterapia é a escolaridade, considerando a compreensão e habilidade, com a terapia complexa ou não. Foi constatado um predomínio de ensino fundamental incompleto/completo (46\%), havendo também analfabetos $(27 \%)$.

A Figura 1 apresenta os problemas de saúde relacionados aos participantes. Alguns dos problemas mais prevalentes na população idosa, foram a hipertensão $(27,20 \%)$ e Diabetes mellitus $(22,70 \%)$. A Figura 2 mostra as classes farmacológicas mais utilizadas, com predominância de anti-hipertensivos (23\%), seguido de anti-diabéticos (12\%).

Automedicação. Os idosos, de acordo com a literatura consultada, utilizavam, em média, dois a cinco medicamentos sob prescrição médica num mesmo período de tratamento medicamentoso. Esses indivíduos por apresentarem um número alto de comorbidades precisam usar um grande número de medicamentos, refletindo o grande número de idosos no país que praticam a automedicação (6).

Para o autor do artigo B (Tabela 2), esta prática no Brasil não se dá apenas com os Medicamenos Isentos de Prescrição (MIP) (Analgésicos, antipiréticos, antidiarréicos, antiesparmódicos, antiácidos, vitaminas e minerais, e relaxantes musculares), mas, também, medicamento com venda sob prescrição (inibidores da bomba de prótons, Inibidores da Enzima Conversora de Angiotenina (IECAs), diuréricos, $\beta$-bloqueadores) e preta (sedativos e hipnóticos). Este último ponto diferindo dos resultados dos demais autores (7).

No estudo descrito no artigo C (Tabela 2) realizado em Salgueiro, PE (2007), foram entrevistados 355 pacientes com média de 60 anos. Os principais motivos que levaram os pacientes à automedicação foram os sintomas de doenças (16\%), tais como hipertensão, diabetes mellitus, e a prescrição médica $(81,5 \%)$. As classes farmacológicas mais utilizadas indicada por presrição médica foram os antihipertensivos $(8,1 \%)$, antidiabéticos $(3,5 \%)$ e ansiolíticos (3,7\%). Sem a prescrição médica os analgésicos $(30 \%)$ e antipirético (29\%) de venda livre, os chamados MIP, foram os principais, de forma semelhante ao estudo descrito no artigo B (7). Tal prática é uma forma de autocuidado, mas que pode constituir um problema à saúde do idoso (8).

No estudo descrito no artigo D (Tabela 2) realizado no em Goiânia, GO (2013), foram entrevistados 934 pacientes com idade média de 71,9 anos (9). Os principais motivos para a automedicação foram os sintomas de doenças $(16,2 \%)$ e indicação médica $(83,7 \%)$. Esta última podendo ser prescrições reutilizadas de indicações passadas, usadas para tratar os mesmos sintomas, sem nova avaliação médica e tais achados foram muito semelhantes áqueles apresentados no artigo C (8). De forma semelhante aos artigos consultados B (7) e C (8), os medicamentos mais utilizados foram os MIP analgésicos/relaxante muscular (30,8\%), seguidos de anti-hipertensivos (14,8\%), fitoterápicos $(7,8 \%)$ e anti-inflamatório (5\%). Ainda, o artigo mostrou uma classe pouca estudada, mas importante na prática da automedicação, os fitoterápicos (9). 


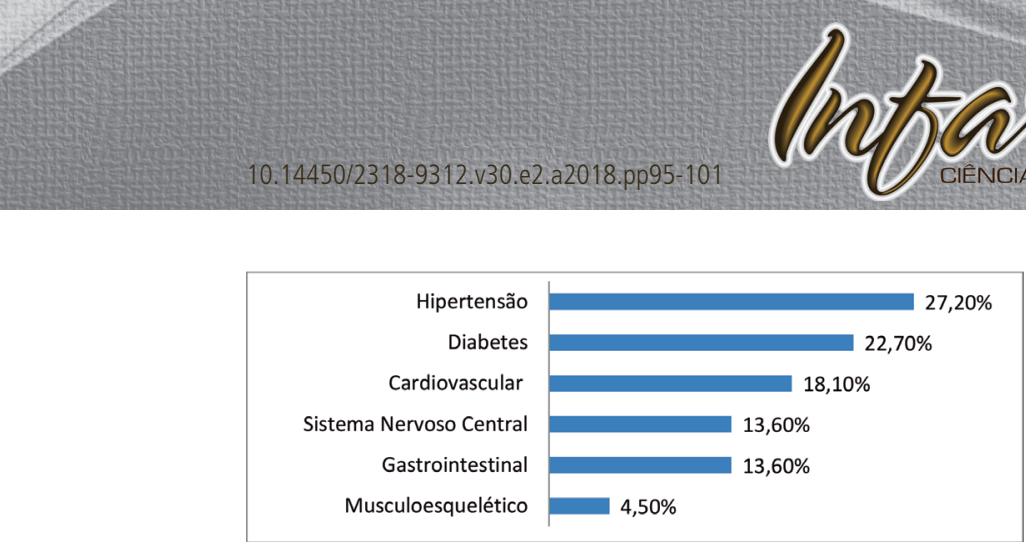

Figura 1. Problemas de saúde existentes nos idosos, de acordo com a literatura consultada (2000-2016)

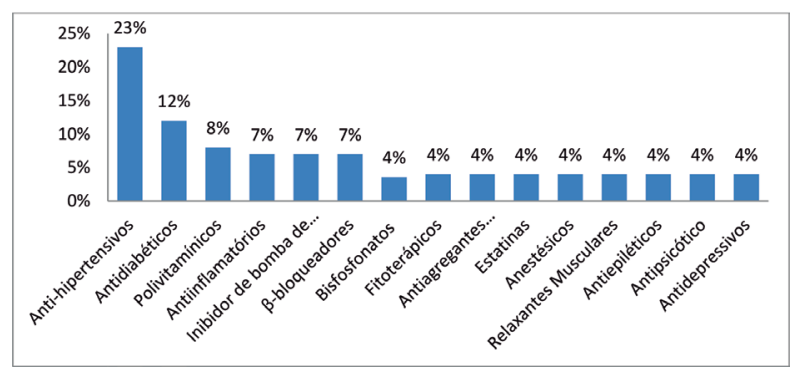

Figura 2. Classes farmacológicas mais utilizadas pelos idosos, de acordo com a literatura consultada (2000-2016)

Polifarmacoterapia. Com relação ao estudo mostrado no artigo E, realizado em Bambuí, MG (2004), foram entrevistados 1.426 pacientes com idade média de 69 anos. Foi constatado que $70 \%$ dos entrevistados estavam submetidos à polifarmacoterapia (10). Ainda, segundo o autor, as doenças crônicas são aquelas que apresentam maior relevância e associação na contribuição para a polifarmácia e um número significativo de medicamentos utilizados nessa prática pode advir da automedicação (10).

Como mostrado no artigo F, realizado em Goiânia, GO (2014), foram assistidos 418 pacientes com idade média de 65 anos (11). Foi mostrado que $72 \%$ dos idosos utilizavam mais de um medicamento, mostrando um resultado percentual semelhante ao artigo A (6). Justificativa para esse resultado, podem ser a baixa frequência de tratamentos não-farmacológicos, o fácil acesso ao medicamento em farmácias comunitária, a tentativa de amenizar sintomas ou as respostas mais rápidas em relação às terapias não-farmacológicas (11).

Como resultado do estudo descrito no artigo A, para cada 10 idosos na faixa etária acima dos 60 anos, 6 estavam sob regime de polifarmácia $(60 \%)$, semelhante percentualmente aos artigos E e F $(10,11)$. Classes farmacológicas como benzodiazepínicos, antigripais, antidepressivos e anti-inflamatórios são consideradas as que mais apresentam possibilidade de intoxicação do usuário, levando a sérias consequências para o idoso, podendo ser fatais devido às alterações no metabolismo, Podem, ainda, levar ao comprometimento de alguns

órgãos e funções, bem como ao comprometimento farmacocinético e farmacodinâmico (6).

Problema Relacionado a Medicamento (PRM). Com relação ao artigo G, o estudo apresentado foi realizado em Florianópolis, SC (2012), no qual foram analisados dados de 58 pacientes com idade média de 62 anos. Foram identificados 157 PRM, sendo a maioria relacionada a subdose $(35 \%)$ e reações adversas (29\%), interferindo diretamente com a efetividade e a segurança, gerando comprometimento da adesão farmacoterapêutica e da saúde do idoso (12).

$\mathrm{O}$ artigo $\mathrm{H}$ apresentou um estudo realizado em Goiânia, GO (2010), no qual foram analisados 50 pacientes com média de idade de 60 anos. Foram identificados 154 PRM, sendo a maioria relacionada a farmacoterapia não efetiva (49\%) e reação adversa (43\%) (13). Estes dois PRM são praticamente idênticos aos encontrados no artigos G e I $(12,14)$, podendo gerar complicações, às vezes, irreversíveis para o idoso.

$\mathrm{O}$ artigo I mostrou um estudo de caso com 4 pacientes idosos com média de 67 anos, portando doenças crônicas, e seus cuidadores, que foi realizado em Florianópolis, SC (2008) (14). Foram detectados PRM ligadas à efetividade (4), à segurança (3), à polifarmacoterapia (2) e à falta de orientação ao cuidador (4). Esse estudo diferiu daqueles descritos nos artigos $\mathrm{G}$ e $\mathrm{H}(12,13)$ quanto a considerar que, na maioria dos casos, os PRM não estavam relacionados apenas ao medicamento, mas também, ao contexto social do indivíduo. Entretanto, tais fatores sociais são, geralmente, excluídos no momento da determinação de causa/diagnóstico de PRM e na formulação de intervenções (14).

O estudo do artigo $\mathrm{J}$ foi realizado em Parobé, RS (2012), onde foram entrevistados 45 pacientes com idade média de 69 anos (15). Em outro estudo (artigo K), realizado em Apucarana, PR (2007), foram entrevistados 71 pacientes com idade média de 67,1 anos (16). No artigo J, foram constatadas 101 interações medicamentosas potenciais, sendo 5 de gravidade maior, 86 moderada e 10 menor ou leve (15), enquanto no artigo $\mathrm{K}$ foram constatadas 69 interações medicamentosas, sendo 6 de gravidade maior, 49 moderada e 19 menor ou leve (16).

Inibidores da enzima conversora da angiotensina (IECA) (enalapril, captopril e ramipril) e diuréticos (furosemida, hidroclorotiazida e indapamida) foi a associação medicamentosa mais envolvida em interações (vinte detectadas) no artigo J, seguidas por outras associações 
como hipogliceminantes (insulina, glibenclamida e metformina) e IECA (treze), hipogliceminantes e diuréticos (onze) e, ainda, IECA e àcido acetilsalicílico (onze). Porém, os fármacos mais envolvidos neste processo são diferentes entre os artigos, à exceção do captopril. Além disso, este foi o fármaco mais envolvido em interação medicamento-alimento (oito), seguido de clorpromazina e biperideno (sete), carbamazepina e haloperidol (sete) $(15,16)$.

Há relação destes resultados com os artigos G, $\mathrm{H}$ e I (12-14), podendo as interações medicamentosas comprometerem tanto a segurança quanto a efetividade, gerando complicações à saúde do idoso. As diferenças observadas quanto às classes farmacológicas envolvidas nas interações medicamentosas podem estar relacionadas ao fato de os estudos terem abordado instituições distintas, tipos de medicamentos utilizados pelos pacientes, bem como a metodologia utilizada.

Adesão à farmacoterapia. No estudo mostrado no artigo L, realizado em Salto Grande, SP (2010), foram entrevistados 102 idosos com média de 69 anos (17). Foi constatado que uma parcela significativa ( $85 \%)$ não aderia adequadamente ao tratamento medicamentoso prescrito e apenas $15 \%$ aderiam de forma satisfatória ao tratamento. Assim, pode ser inferido que estes pacientes estavam predispostos a complicações das doenças crônicas, gerando maiores gastos com serviços de saúde, tanto no serviço público de saúde quanto na sua própria renda (17).

O estudo mostrado no artigo $\mathrm{M}$ foi realizado em Solonópole, $\mathrm{CE}$, onde foram entrevistados 33 idosos com média de 68 anos portadores de doenças crônicas (18) e a maioria dos pacientes (64\%) não aderia corretamente à farmacoterapia, o que pode ser justificado por uma maior dificuldade na utilização do medicamento, menor habilidade para apreender e adquirir novos domínios e informações (18). No estudo apresentado no artigo N, também desenvolvido no Nordeste, foi observada uma maior adesão ao medicamento (19). Em contrapartida, os resultados das avaliações citadas nos artigos L e M $(16,17)$ foram praticamente semelhantes quanto ao percentual, com predominância de pacientes que não aderiram ao tratamento.

Em estudo referente ao artigo N, realizado em São Luís, MA (2015), foram entrevistados 308 idosos com média de 63 anos e doenças crônicas (19). Diferente dos resultados mostrados nos artigos L e M $(16,17)$, foi constatado que a maioria mostrava aderência ao tratamento medicamentoso $(60,6 \%)$, e foram apontados, como fatores relevantes e facilitadores dessa adesão, o apoio da família no processo de cuidado, a necessidade de cumprimento da farmacoterapia, o medo do agravo â saúde e a vontade de viver (19).

\section{CONCLUSÃO}

Foram constatados problemas significativos em vários aspectos da farmacoterapia, como polifarmacoterapia, automedicação, PRM, intervindo na adesão farmacoterapêutica.

Os resultados deste estudo possibilitam depreender a praticamente inexistência da prática da atenção farmacêutica em algumas localidades do país, ou seja, idosos estão desassistidos com relação à farmacoterapia, tornando-os expostos a problemas relacionados a medicamentos e/ou agravamento de doenças já existentes.

A atenção farmacêutica, no contexto de problemas de saúde em idosos, é essencial para o fortalecimento da adesão ao tratamento, redução de gastos, redução de PRM, e disponibilização de informações sobre a doença e seus agravos.

Portanto, as informações sobre medicamentos, juntamente com as habilidades de comunicação, são as principais ferramentas que o farmacêutico deve explorar durante sua prática clínica, oportunidade, também, para consolidação desta área de atuação profissional. 


\section{REFERÊNCIAS}

1. IBGE. Instituto Brasileiro de Geografia e Estatística 2010. [citado em 19 de fevereiro de 2017]. Disponível em: http://www.ibge.gov.br/apps/populacao/projecao.

2. Souza RR. O sistema público de saúde brasileiro. Brasília: Ministério da Saúde, 2002.

3. Porth CM. Matfin, G. Conceitos de saúde alterada em adultos idosos. In: Fisiopatologia. Rio de Janeiro: Editora Guanabara Koogan. 2010. p.38-58.

4. BRASIL. Ministério da Saúde. Portaria $n^{\circ} 2.488$, de 21 de outubro de 2011. Aprova a Política Nacional de Atenção Básica, estabelecendo a revisão de diretrizes e normas para a organização da Atenção Básica, para a Estratégia Saúde da Família (ESF) e o Programa de Agentes Comunitário de Saúde (PACS). Diário Oficial da União, nº 63, 24 de outubro de 2011. Seção 1. p. 48.

5. BRASIL. Ministério da Saúde. Resolução no 338, de 6 maio de 2004. Aprova a Política Nacional de Assistência Farmacêutica. Diário Oficial da União, $n^{\circ}$ 8, 07 de maio 2004. Seção 1. p. 64.

6. Bortolon PC, Karnikowski MGO, Assis M. Automedicação versus indicação farmacêutica: o profissional de farmácia na atenção primária à saúde do idoso. Revista APS. 2007;10(2):200-209.

7. Fernandes WS, Cembranelli JC. Automedicação e o uso irracional de medicamentos: o papel do profissional farmacêutico no combate a essas práticas. Rev. Univap. 2015;21(37):05-12. DOI: 10.18066/revistaunivap. v21i37.265.

8. Sá M, Barros JAC, Sá MPB. Automedicação em idosos na cidade de Salgueiro-PE. Rev. Bras. Epidemiol. 2007;10(1):75-85. DOI: $10.1590 / \mathrm{S} 1415-$ 790X2007000100009.

9. Santos TRA, Lima DM, Nakatani AYK, Pereira LV, Leal GS, Amaral RG. Consumo de medicamentos por idosos. Rev. Saúde Pública. 2013;47(1):94-103. DOI: 10.1590/ S0034-89102013000100013.

10. Lima-Costa MF, Firmo JOA, Uchoa EA. A estrutura da auto-avaliação da saúde entre idosos: projeto Bambuí. Rev Saúde Pública. 2004;38(6):827-34. DOI: 10.1590/ S0034-89102004000600011.
11. Silveira EA, Dalastra L, Pagotto V. Polifarmácia, doenças crônicas e marcadores nutricionais em idosos. Rev. Bras. Epidemiol. 2014;17(4):818-829. DOI: 10.1590/18094503201400040002.

12. Alano GM, Corrêa TS, Galato D. Indicadores do serviço de atenção farmacêutica (SAF) da Universidade do Sul de Santa Catarina. Cien \& Saúde Colet. 2012;17(3):757764. DOI: $10.1590 / \mathrm{S} 1413-81232012000300023$.

13. Provin MP, Campos AP, Neilson SEO, Amaral RG. Atenção farmacêutica em Goiânia: inserção do farmacêutico na estratégia saúde da família. Saúde Soc. 2010;19(3):717-723. DOI: 10.1590/S010412902010000300022.

14. Foppa AA, Bevilacqua G, Pinto HL, Blatt CR. Atenção farmacêutica no contexto da estratégia de saúde da família. Rev. Bras. Cien. Farm. 2008;44(4): 728-737. DOI: $10.1590 / \mathrm{S} 1516-93322008000400020$.

15. Amaral DMD, Perassolo MS. Possíveis interações medicamentosas entre os anti-hipertensivos e antidiabéticos em participantes do Grupo HIPERDIA de Perobé, RS (Uma análise teórica). Rev. Farm. Básica e Apl. 2013;33(1):99-105.

16. Correr CJ, Pontarolo R, Ferreira LC, Baptistão SAM. Risco de problemas relacionados com medicamentos em pacientes de uma instituição geriátrica. Rev. Bras. Ciênc. Farm. 2007;43(1):56-62. DOI: 10.1590/S151693322007000100007.

17. Obreli-Neto PR, Prado MF, Vieira JC, Pelloso SM, Marcon SS, Cuman RKN. Fatores interferentes na taxa de adesão à farmacoterapia em idosos atendidos na rede pública de saúde do Município de Salto Grande-SP, Brasil. Rev. Ciênc. Farm. Básica e Apl. 2010;31(3):229233.

18. Landim MP, Oliveira CJ, Abreu RNDC, Moreira TMM, Vasconcelos SMM. Adesão ao tratamento farmacológico anti-hipertensivo por pacientes de unidade da estratégia saúde da família. Rev. APS. 2011;14(2):132-138.

19. Sardinha AHL, Silva CG, Sena LB, Mesquita LLS, Rodrigues JB, Silva KNR. Adesão dos idosos com doenças crônicas ao tratamento medicamentoso. Rev. Pesq. Saúde. 2015;16(3):154-158. 